\title{
Evaluating the Hydrodynamic Response of Coastal Bridges during an Extreme Weather Event
}

\author{
Reza Nasouri ${ }^{1}$, Adnan Shahriar ${ }^{2}$, Adolfo Matamoros ${ }^{1}$, Arturo Montoya $^{1,{ }^{*}}$, and First Testik ${ }^{1}$ \\ ${ }^{1}$ Department of Civil and Environmental Engineering, University of Texas at San Antonio, San Antonio, TX 78249 \\ ${ }^{2}$ Department of Mechanical Engineering, University of Texas at San Antonio, San Antonio, TX 78249
}

\begin{abstract}
The frequency and intensity of recent hurricanes have demonstrated the need of taking proactive actions to prevent major damages during an extreme weather event. This work presents the results of a numerical study evaluating the hydrodynamic response of coastal bridges during an extreme hurricane event. A finite element model of a concrete bridge girder superstructure with a pier-substructure was developed in the commercial software Abaqus. The Coupled Eulerian-Lagrangian technique was used to model the interaction between water waves and the bridge as the structure deformed due to wave impacts. The wave velocity and the angle of wave impact were varied in the simulation to determine their effects on the response of the bridge. It was found that the resultant shear and uplift forces increase with wave velocity, while the angle of impact only had a significant effect on the resultant shear forces. The developed numerical framework will support further studies that will investigate variations in the bridge design and construction practices in order to enhance the resilience of coastal bridges against extreme weather events.
\end{abstract}

\section{Introduction}

The strategies adopted to cope with extreme hurricane and tsunami events in the United States have been reactive rather than proactive. Padgett et al. [1] indicated that the cost of repairing and replacing bridges that were damaged during hurricane Katrina exceeded 1 billion US dollars. The most severe damage consisted of superstructure collapse due to unseating of the deck, caused by the combined actions of storm surge and waves. This type of failure was observed both in bridges with integral and non-integral supports. In some instances, uplift forces were large enough to exceed the weight of the superstructure and cause the failure of the connection at the support. It was also observed that in some cases, shear keys were sufficient to prevent unseating of the superstructure.

Laboratory tests have been conducted in order to have a better understanding of the effects of wave loadings on structures. Bradner et al. [2] conducted a test on a 1:5 scale concrete bridge superstructure section under hurricane wave loads and Linton et al. [3] performed a full-scale test on a light frame wood shear wall under tsunami load. Both experiments indicated that dynamic loads are greater in magnitude than quasi-static loads. However, due to the cost and time consumption associated with laboratory testing, research has shifted towards analyzing the effects of hydrodynamic loads on structures through computational approaches. Bozorgnia et al. [4] used a computational fluid dynamics (CFD) approach to study the effects of entrapped air on wave impact and uplift forces on a 2D model of a bridge section. Chen et al. [5] investigated the wave structure interaction using a popular CFD code, OpenFOAM [68].

A recent performance-based approach to determine the reliability of elevated coastal structures subjected to wave loads was conducted by Do et al. [9]. They used the Coupled Eulerian-Lagrangian (CEL) approach, available in the commercial finite element software ABAQUS [10], to compute forces on a girder bridge model. The wave loading input to the CEL simulation was generated based on a power spectrum density (PSD) function dependent on two sea state variables, significant wave height and peak period. The PSD variables were determined based on historical data. The simulation consisted of a 300-second period in which several waves were generated according to the PSD. The probability of failure of the bridge model was calculated by finding the ratio of the number of waves that generated loads that exceeded the uplift capacity of the bridge to the total number of wave events. Do et al. [9] repeated the simulation for several combinations of significant wave height and peak wave period. Then, these simulation outputs were used to develop fragility curves that indicate the probability of failure under several sea states. More recently, Yuan et al. [11] proposed an ultimate resistance envelope for evaluating the performance of connections between the bridge superstructure and substructure. $\mathrm{Qu}$ et al. [12] demonstrated through numerical simulations that submersion depth influences the vertical component of the hydrodynamic load.

\footnotetext{
$\overline{\text { * Corresponding author: arturo.montoya } @ \text { utsa.edu }}$
} 
The objective of this study is to evaluate the hydrodynamic response of a coastal bridge due to wave impacts with different characteristics, i.e. velocity and angle of impact, induced during extreme weather events.

\section{Methodology}

The Coupled Eulerian-Lagrangian (CEL) technique available in Abaqus was used to model the FluidStructure Interaction (FSI). In the CEL technique, solids are simulated with Lagrangian meshes, while fluids are simulated using Eulerian meshes. Lagrangian meshes are attached to material points, and as the materials deform, the mesh deforms with them. Eulerian meshes remain the same as the material flows (or deforms) within the mesh, acting as a background grid [13]. Although CEL simulations are computational costly, they can provide a full description of the stress field acting on the bridge members due to wave impacts with acceptable accuracy. This detailed analysis cannot be achieved with basic equations in existing code manuals. The stresses acting on a surface can be integrated in order to determine the lateral and uplift loads generated throughout the simulation history.

In order to verify the appropriate use of the CEL technique, the authors replicated the numerical simulation presented by Do et al. [9], consisting of a 1:5 scale reinforced concrete bridge super structure model subjected to hurricane waves. The results of this numerical simulation were validated by comparing the uplift forces obtained from the simulation with the experimental results performed by Bradner et al. [2]. Figure 1(a) illustrates the bridge superstructure model, having a length of $1.94 \mathrm{~m}$, width of $3.45 \mathrm{~m}$, and the volume of the sea water material $(20 \mathrm{~m} \times 6 \mathrm{~m} \times 2 \mathrm{~m})$. The superstructure has material properties corresponding to a $40 \mathrm{MPa}$ concrete.

The boundary conditions of the simulation are shown in Figure 1(b). The input velocity at the left face is altered to generate wave elevations of $0.5 \mathrm{~m}$ and time periods of 2 seconds as observed in the experiment. At the right end, the pressure is set to zero in order to replicate outlet conditions. The velocity at the bottom face is specified as zero to satisfy the no-slip boundary condition. At the side walls, water velocity component perpendicular to the walls is set to zero and velocity components parallel to the walls were not subject to wall shear (i.e. slip boundary condition). The front support of the superstructure is restrained in the $\mathrm{x}$ and $\mathrm{y}$ direction, while the rear of the concrete structure has fixed support conditions.

Figure 2 compares the uplift loads obtained by the numerical simulation and the experimental results. This comparison shows a close agreement between the numerical and experimental results; particularly, in capturing the ascending and descending trend of the uplift force. The magnitudes of the uplift forces are dependent on the flexibility of the substructure. This model assumes rigid substructure conditions, which generates large uplift forces in order to ensure the boundary conditions of the model. Neglecting the ability of the substructure to deform may result in inaccurate stress demand predictions and eventually lead to an overdesign of the bridge structural members and connections. Thus, in this study, a pier-substructure was added to the model in order to determine its effect on the response of the structure.

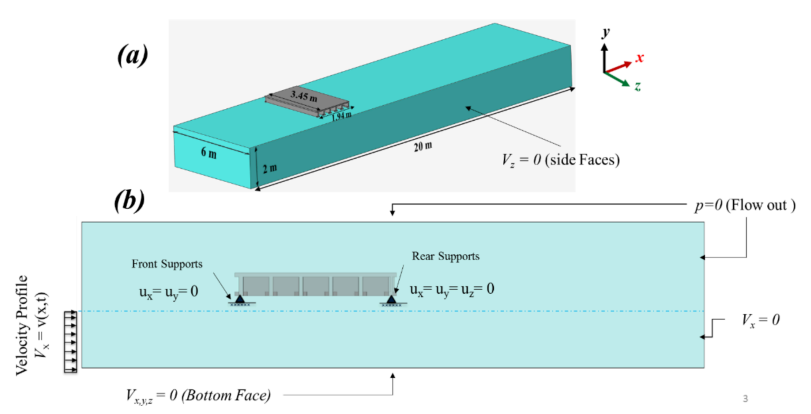

Fig. 1. (a) Bridge verification model and (b) corresponding boundary conditions.

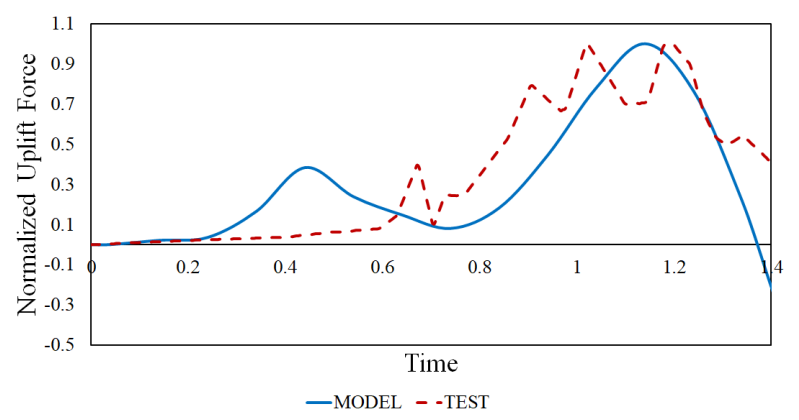

Fig. 2. Comparison of the uplift forces between the numerical model and the experimental test for the verification study.

\subsection{Finite element models}

A bridge model with a pier substructure was developed as shown in Figure 3. The superstructure conditions described in the verification study were kept constant. The concrete piles have a diameter of $24 \mathrm{~cm}$ and are $2 \mathrm{~m}$ long. In between the substructure and the piers, there are pier caps of rectangular cross-section placed along the width of the superstructure (x-direction of the model). The concrete caps have a cross-section of $30 \mathrm{~cm} \times 10$ $\mathrm{cm}$.

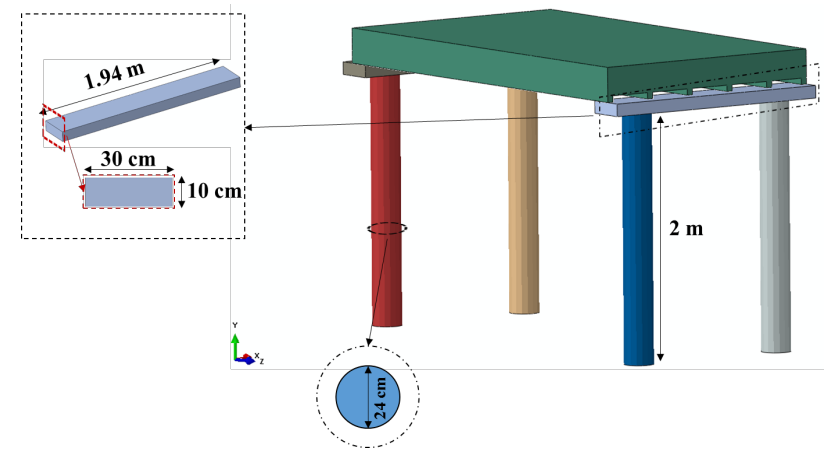

Fig. 3. Bridge model. 
The boundary conditions of the simulations are illustrated in Figure 4. The piers were provided fixed boundary conditions at the bottom surface. Tie connections were used to simulate the interaction between the pier caps and the piers, and between the end supports of the bridge superstructure with the pier caps. The contact between the interior beams of the superstructure and the pier caps was modeled by using a friction model.

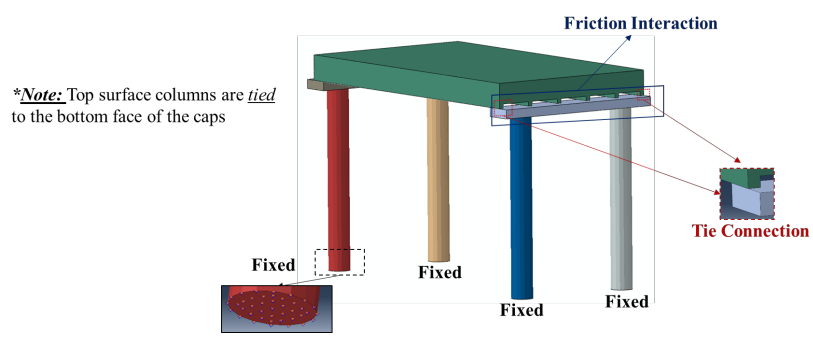

Fig. 4. Boundary conditions for the bridge models with pier substructures.

The amplitude and period of the generated waves were varied by altering the velocity input in the models. In addition, as the waves can impact the bridge at different angles, the angle of wave impact was varied in the simulations. This variation was implemented numerically by keeping the water flow along the $\mathrm{x}$ direction, but rotating the orientation of the bridge by $15^{\circ}, 30^{\circ}$, and $45^{\circ}$ as shown in Figure 5. The $0^{\circ}$ model corresponds to the case in which the wave impact is perpendicular to the bridge deck. The wave velocity was kept at $1 \mathrm{~m} / \mathrm{s}$ for all rotated models.

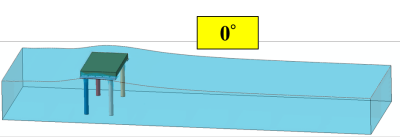

(a)

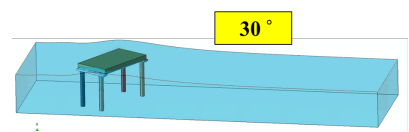

(c)

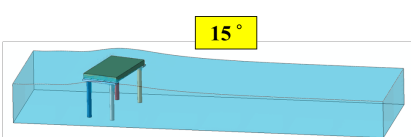

(b)

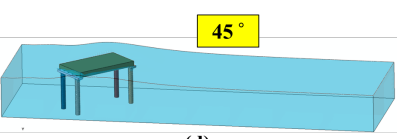

(d)
Fig. 5. Bridge orientations against water flow along the $x$ direction

\section{Results}

Figure 6 shows the displacements along the direction of water flow (x-direction) for different wave velocities. The open circle at the superstructure midspan indicates the location in which the displacements were extracted for plotting. This plot indicates that there was an incrementally proportional correspondence between wave velocity and displacements. The period of oscillation of the bridge is about 3 seconds. The impact force generated on the bridge is proportional to the magnitude of the water velocity. Figure 7 illustrates the von Mises stress at the end support of the bridge superstructure with the pier cap, indicating that stress demands are proportional to the magnitude of wave impacts. The stresses at the lower surface of the superstructure were integrated in order to determine the resultant shear and uplift forces acting at the interface between the superstructure and the pier cap throughout the 10 seconds of the simulation. Figure 8 plots the shear (force along the x-direction) and uplift forces for each of the wave velocities under consideration. The uplift forces were found to be significantly higher in magnitude than the shear forces, having a peak of $40 \mathrm{kN}$ as compared to the maximum shear force of $6 \mathrm{kN}$.
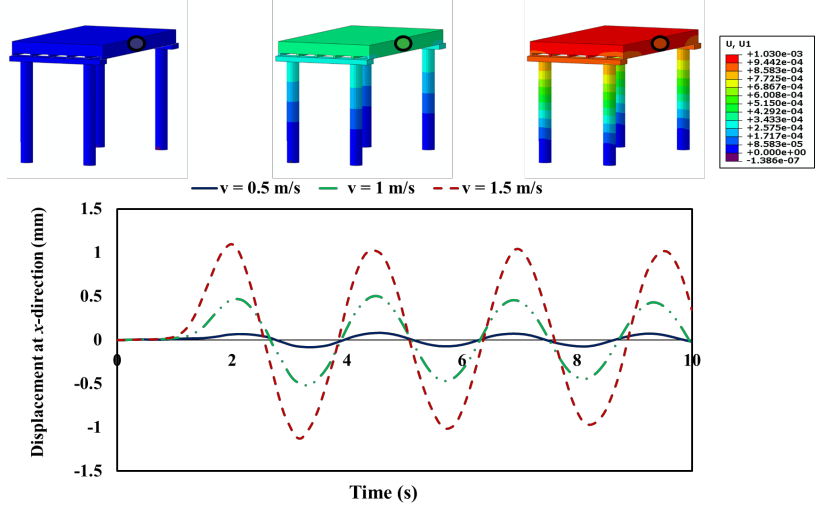

Fig. 6. Displacements along the x-direction for Model 1 under three different wave velocities.

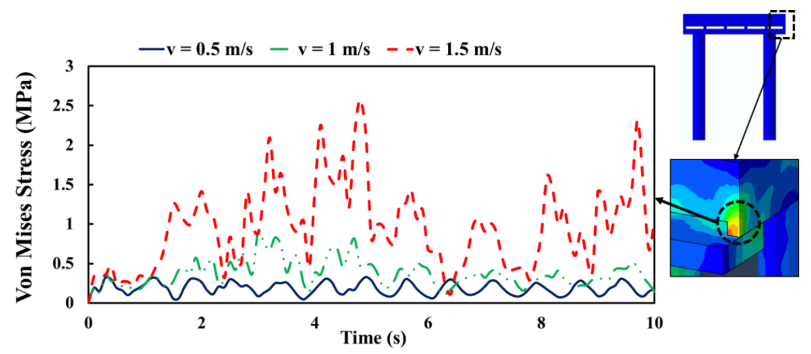

Fig. 7. Von Miss Stress for Model 1 under three different wave velocities.

Figure 9 shows the resultant forces normalized by the bridge self-weight for the models that were rotated in order to account for the variations in the angle of wave impact. It can be observed that the angle of wave impact had a negligible effect on the uplift force, but varied the magnitude of the shear force components along the $\mathrm{x}$ (along the water flow) and $\mathrm{z}$-(perpendicular to the water flow) directions. The force in the $\mathrm{x}$-direction for the wave impacts at $30^{\circ}$ and $45^{\circ}$ angles increased considerably as compared to the lower angle results, $0^{\circ}$ and $15^{\circ}$. The force in the $\mathrm{z}$-direction for higher angles had a larger magnitude immediately after impact, but then lowered down throughout the simulation as compared to the smaller angles. 


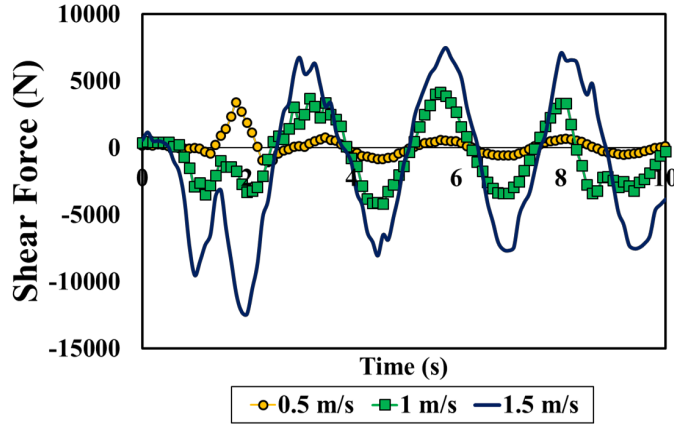

(a)

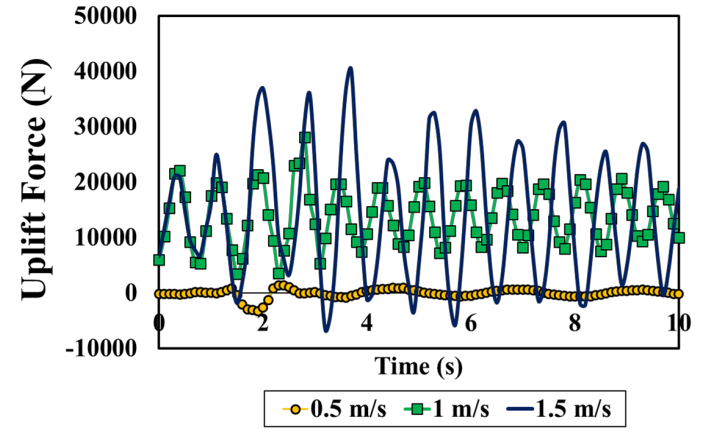

(b)

Fig. 8. (a) Shear and (b) uplift force acting on the concrete superstructure.
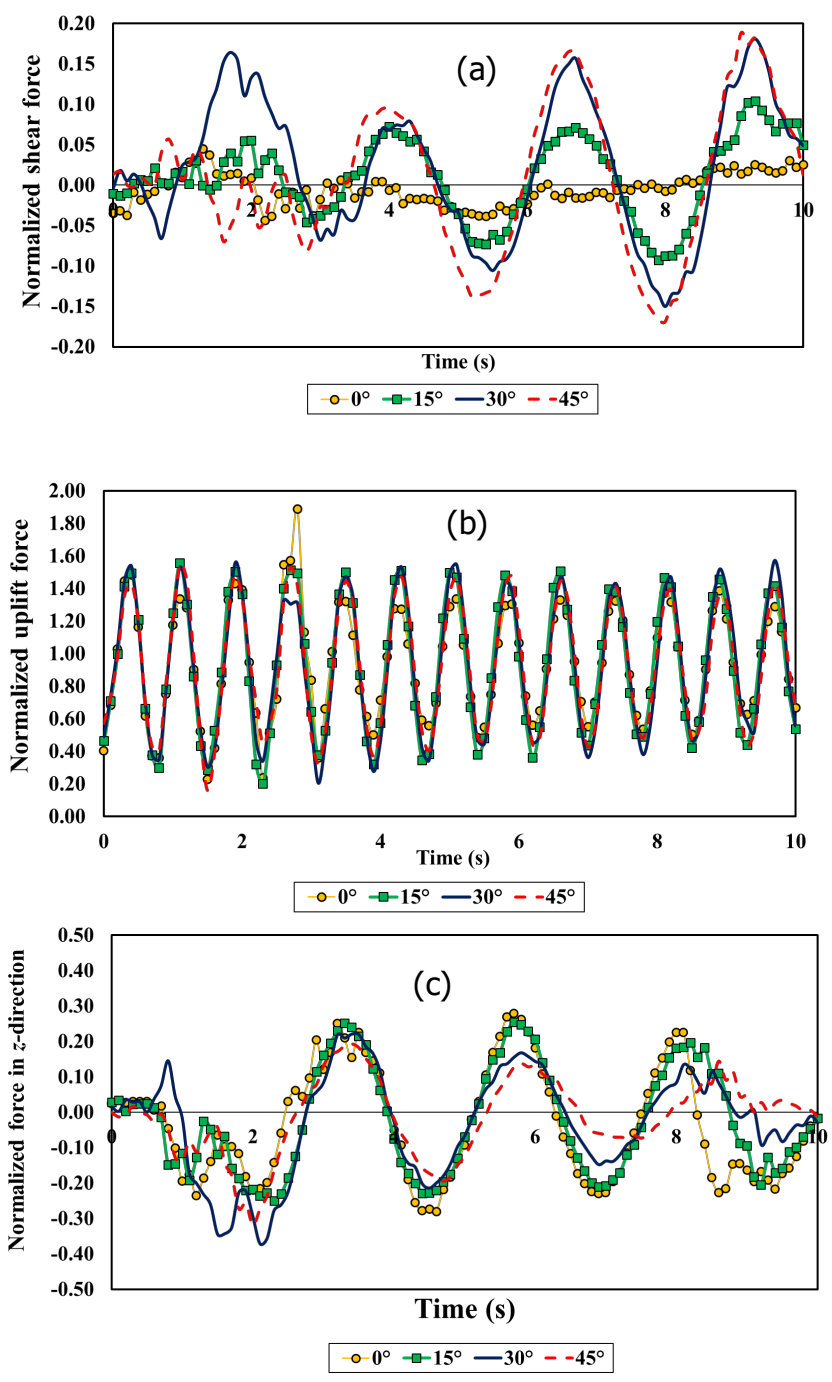

Fig. 9. Resultant force components along the (a) $x$-direction (b) $\mathrm{y}$-direction (uplift force) and (c) z-direction acting on the concrete superstructure

\section{Conclusions}

In this research work, the response of a coastal bridge, with a concrete superstructure and pier-substructure, under the impact of hurricane induced waves was simulated numerically using the Abaqus software and the CEL technique. The simulation results indicated that the response of the structure is highly dependent on the wave characteristics. The resultant shear and uplift forces increased with wave velocity, while the angle of impact only varied the magnitude of the resultant shear forces. In future work, variations to the bridge configuration will be performed in order to obtain a better understanding of the response of coastal bridges under hydrodynamic loads and propose design modifications that make them more resilient to extreme weather events.

\section{References}

1. Padgett, J., DesRoches, R., Nielson, B., Yashinsky, M., Kwon, O.S., Burdette, N., and Tavera, E. (2008). Bridge damage and repair costs from Hurricane Katrina. Journal of Bridge Engineering, 13(1), 6-14.

2. Bradner, C., Schumacher, T., Cox, D., and Higgins, C. (2010). Experimental setup for a large-scale bridge superstructure model subjected to waves. Journal of waterway, port, coastal, and ocean engineering, 137(1), 3-11.

3. Linton, D., Gupta, R., Cox, D., van de Lindt, J., Oshnack, M.E., and Clauson, M. (2012). Evaluation of tsunami loads on wood-frame walls at full scale. Journal of Structural Engineering, 139(8), 1318-1325.

4. Bozorgnia, M., Lee, J.J., and Raichlen, F. (2011). Wave structure interaction: Role of entrapped air on wave impact and uplift forces. Coastal Engineering Proceedings, 1(32), 57.

5. Chen, L., Zang, J., Hillis, A., Morgan, G., and Plummer, A. (2014). Numerical investigation of wave-structure interaction using OpenFOAM. Ocean Engineering, 88, 91-109.

6. N. Asadollahi Shahbaboli (2016). Numerical Modeling of Extreme Flow Impacts on Structures. Université d'Ottawa/University of Ottawa.

7. Bricker, J.D., Kawashima, K., and Nakayama, A. (2011). CFD analysis of bridge deck failure due to tsunami. Proc., Proceedings of the international symposium on engineering lessons learned from the, 1-4.

8. Weller, H. (2010). OpenFOAM: The Open Source CFD Toolbox User Guide, Version 2.1.0.

9. Do, T.Q., van de Lindt, J.W., and Cox, D.T. (2016). Performance-based design methodology for 
inundated elevated coastal structures subjected to wave load. Engineering Structures, 117, 250-262.

10. Simulia, A.V. (2013). 6.13 Documentation. Dassault systemes.

11. Yuan, P., Xu, G., Chen, Q., and Cai, C.S. (2018). Framework of Practical Performance Evaluation and Concept of Interface Design for Bridge DeckWave Interaction. Journal of Bridge Engineering, 23(7), 04018048.

12. Qu, K., Tang, H.S., Agrawal, A., Cai, Y., and Jiang, C.B. (2018). Numerical investigation of hydrodynamic load on bridge deck under joint action of solitary wave and current. Applied Ocean Research, 75, 100-116.

13. Durst, F., Miloievic, D., and Schönung, B. (1984). Eulerian and Lagrangian predictions of particulate two-phase flows: a numerical study. Applied Mathematical Modelling, 8(2), 101-115. 